\title{
Aortic valve replacement due to lactococcus lactis infective endocarditis
}

\author{
Özgür Altınbaş ${ }^{1}$, Erdal Ege $^{2}$, Ali Sarıgül ${ }^{2}$
}

1) Necmettin Erbakan University, Meram Medical Faculty, Department of Cardiovascular Surgery, Ass. Dr., Konya, Turkey

${ }^{2)}$ Necmettin Erbakan University, Meram Medical Faculty, Department of Cardiovascular Surgery, Prof. Dr., Konya, Turkey

\begin{abstract}
Infective endocarditis characterized by microbial infection of the endothelial surface of the heart,has an estimated annual incidence of 3 to 9 cases per 100.000 persons in industralized countries. Although common species causing infective endocarditis include streptococci, staphylococci, enterococci and fastidious gram negative coccobacilli, aortic valve replacement due to lactococcus lactis infective endocarditis seen in the literature, even if rarely. In this study we presented a 34 year old male patient underwent surgery, diagnosed with lactococcus lactis infective endocarditis.
\end{abstract}

Keywords: Infective endocarditis, Lactococcus lactis, Complication 


\section{Introduction}

Endovascular, microbial infection of intracardiac structures facing the blood including infections of the large intrathoracic vessels and of intracardiac foreign bodies, called infective endocarditis. The early characteristic lesion is a different sized vegetation, although destruction, ulceration or abscess formation may be seen earlier by echocardiography. ${ }^{(1)}$

The highest rates of the infective endocarditis are observed among patients with prosthetic valves, intracardiac devices, unrepaired cyanotic congenital heart diseases or a history of infective endocarditis, although $50 \%$ of cases of infective endocarditis develop in patients with no known history of valve disease. Other risk factors include chronic rheumatic heart disease, age-related degenerative valvular lesions, hemodialysis and coexisting conditions such as diabetes, human immunodeficiency virus infection and intravenous drug use. Diagnosis of endocarditis is usually based on clinical, microbiologic and echocardiographic findings. ${ }^{(2)}$

Indications for cardiac surgery are; heart failure, no control of infection, vegetations and embolic risk, perivalvar infection, valvar obstruction, unstable prosthesis, prosthetic infective endocarditis, fungal infective endocarditis, difficult-to-treat microorganisms and neurological complications. Lactococcus lactis is a mesophilic and microaerophilic fermenting bacteria, used for fermented food products production. It can be isolated even if rarely from oropharinx, intestines, or vagina as a part of normal flora. For a long time it was considered as nonvirulent with low pathogenity in humans. ${ }^{(3)}$

\section{Case Report}

Thirty four year old male patient, previously healthy, sometimes presented with a high fever, cold and chills problems last one mounth. He had first applied to a health care center and oral antibiotherapic mediaction was started but his problems had continued to exist. And than patient was applied to a hospital and though there was a suspicious aspect in transthorasic echocardiography, he was referred to university hospital for transesophageal echocardiography. He was hospitalized in infectious diseases service and there was no signicant pathology in his physical examination. Four blood cultures were taken from patient and lactococcus lactis was seen in one of them. $1,5 * 2,1 \mathrm{~cm}$ sized vegetations on the aortic valve were determined in transesophageal echocardiography. Ejection fraction was $60 \%$. Aortic regurgatition was $2-3$ rd degree. Intravenous antibiotherapy included gentamicin and vancomycin started to the patient.

The patient was referred to us and because of the risk of embolisation due to vegetation, emergency operation was decided for him. He was interned to cardiovascular intensive care unit and aortic valve replacement was made with 25 no SJ prosthetic valve.Aortic valve wall was fibrocalcific and approximately 1,6*2,2 cm sized vegetations was seen on the valve. There was no microorganism produced in valve culture.

\section{Discussion}

The incidence of IE continues to rise, with a yearly incidence of $\approx 15000$ to 20000 new cases. Although advances in antimicrobial therapy and the development of better diagnostic and surgical techniques have reduced the morbidity and mortality of infective endocarditis, it remains a potentially life-threatening disease. ${ }^{(4)}$ The most common cause of the endocarditis is the infection and endocarditis due to lactococcus lactis is a rare clinical situation that most frequently occurs in immunocompromised patients or in those with impaired local defense mechanism in which this usually non-pathogen microorganism may be cause of severe infection. ${ }^{(5)}$

Diagnostic work-up, including a complete transthoracic and transesophageal study, must be performed immediately in every patient admitted to an intensive care unit with embolism, heart failure, cardiogenic or septic shock of unknown cause, as the data presented here suggest that prompt surgical intervention can be life-saving in patients with infective endocarditis despite the presence of severe shock and the occurrence of multiorgan failure. ${ }^{(6)}$ Infective endocarditis caused by lactococcus lactis is a rare clinical situation,so it must be considered as one of the factors of infective endocarditis. Early surgical intervention in lactococcus lactis endocarditis can save lives. 


\section{References}

1. Dieter Horstkotte,Ferenc Follath,Erno Gutschik,Maria Lengyel,Ali Oto, Alain Pavie et al.Guidelines on Prevention,Diagnosis and Treatment of Infective Endocarditis Executive Summary:The Task Force on Infective Endocarditis of the European Society of Cardiology.Eur Heart J (2004) 25 (3):267-276

2. Bruno Hoen,Xavier Duval.Infective Endocarditis.N Engl J Med 2013;368:1425-1433

3. Bshara Mansour,Adib Habib,Nazih Asli,Yuval Geffen,Dan Miron,Nael Elias.A Case of Infective Endocarditis and Pulmonary Septic Emboli Caused by Lactococcus Lactis.Case Report in Pediatrics. Volume 2016,Article ID 1024054,4 pages
4. Diagnosis and Managment of Infective Endocarditis and its Complications. Arnold S.Bayer,MD;Ann F.Bolger MD;Kathryn A.Taubert,Ph,et al Circulation 1998;98:2936-2948

5. C.Rostagno,P.Pecile,P.L.Stefano.Early Lactococcus lactis endocarditis after mitral valve repair:a case report and literature review.Infection (2013)41:897-899

6. Complicated infective endocarditis necessitating ICU admission: Clinical corse and prognosis

7. Georg Delle Karth,Maria Koreny,Thomas Binder...et al. Critical Care 2002,6:149-154

Received: $12 / 01 / 2017$

Accepted: 04/05/2017

Published: 15/06/2017

Disclosure and conflicts of interest:

Conflicts of interest were not reported.

\section{Corresponding author:}

Dr. Özgür Altınbaş

Mail: ozgurinterpol@yahoo.com 